The Trauma Registry Compared to All Patient Refined Diagnosis Related Groups (APR DRG)

Jodi Hackworth, MPH, CSTR, CAISS ${ }^{1}$

Johanna Askegard-Giesmann, MD, FACS ${ }^{2}$

Thomas Rouse, MD, FACS ${ }^{1,3}$

Brian Benneyworth, MD, MS 4,5

1. Department of Trauma Services, Riley Hospital for Children at IU Health, Indianapolis, IN

2. Department of Surgery, Sanford Health, Fargo, ND

3. Department of Surgery, Indiana University School of Medicine, Indianapolis, IN

4. Section of Pediatric Critical Care Medicine, Department of Pediatrics, Indiana University School of Medicine, Indianapolis, IN

5. Children’s Health Services Research, Indiana University School of Medicine, Indianapolis, IN

Correspondence and Reprints:

Jodi Hackworth, MPH, Riley Hospital for Children at Indiana University Health, 705 Riley Hospital Drive, Suite 1960, Indianapolis, IN 46202. Telephone: 317-944-5434; Fax 317-9446535; E-mail: jhackworth@iuhealth.org.

Acknowledgements:

Source of funding: No funding was received.

Conflicts of Interest:

We have no conflicts of interest to disclose.

This is the author's manuscript of the article published in final edited form as:

Hackworth, J., Askegard-Giesmann, J., Rouse, T., \& Benneyworth, B. (2017). The trauma registry compared to All Patient Refined Diagnosis Groups (APR-DRG). Injury, 48(5), 1063-1068.

https://doi.org/10.1016/j.injury.2016.12.026 


\section{The Trauma Registry Compared to All Patient Refined Diagnosis Related Groups (APR DRG)}

\section{Abstract \\ Background:}

Literature has shown there are significant differences between administrative databases and clinical registry data. Our objective was to compare the identification of trauma patients using All Patient Refined Diagnosis Related Groups (APR-DRG) as compared to the Trauma Registry and estimate the effects of those discrepancies on utilization.

\section{Methods:}

Admitted pediatric patients from 1/2012-12/2013 were abstracted from the trauma registry. The patients were linked to corresponding administrative data using the Pediatric Health Information System database at a single children's hospital. APR-DRGs referencing trauma were used to identify trauma patients. We compared variables related to utilization and diagnosis to determine the level of agreement between the two datasets.

\section{Results:}

There were 1942 trauma registry patients and 980 administrative records identified with traumaspecific APR-DRG during the study period. Forty-two percent (816/1942) of registry records had an associated trauma-specific APR-DRG; 69\% of registry patients requiring ICU care had trauma APR-DRGs; 73\% of registry patients with head injuries had trauma APR-DRGs. Only $21 \%$ of registry patients requiring surgical management had associated trauma APR-DRGs, and $12.5 \%$ of simple fractures had associated trauma APR-DRGs.

\section{Conclusion:}


APR-DRGs appeared to only capture a fraction of the entire trauma population and it tends to be the more severely ill patients. As a result, the administrative data was not able to accurately answer hospital or operating room utilization as well as specific information on diagnosis categories regarding trauma patients. APR-DRG administrative data should not be used as the only data source for evaluating the needs of a trauma program.

Keywords: trauma, administrative data, registry

\section{Background}

As the landscape of healthcare continues to adapt to an environment focused on quality improvement and clinical outcomes, there is an increasing need for readily accessible data related to patient outcomes, healthcare costs, and resource utilization ${ }^{1,2,3}$. Administrative financial data are widely available, use information that is already collected for billing purposes, and are generated from discharge reports submitted to payers. Consequently, administrative datasets are often utilized by hospital leadership to assess quality and performance. These data are subject to limitations of national coding rules and guidelines ${ }^{2,4,5}$. Previous studies have demonstrated that utilizing administrative billing datasets may not identify patients correctly ${ }^{5,6,7}$.

Hospitals designated as trauma centers are required to have trauma registries that contain detailed data on all trauma patients cared for at that specific hospital. Trauma registry data are abstracted from the medical record by registrars using specialized software and specific training ${ }^{8}$. The data collected include information on patient demographics, the circumstances surrounding injury, pre-hospital care, transport, emergency department and inpatient procedures, anatomic injury descriptions, physiological measurements, complications, comorbidities, and outcomes. Trauma registry abstraction differs from administrative coding in that descriptions of injuries and 
medical care are not confined to International Classification of Disease (ICD) groups. In contrast to administrative coding, nursing documentation, laboratory data, and imaging reports can be utilized for registry collection. Wynn et. al. demonstrated that compared to ICD-9 codes in administrative data, trauma registries recorded more diagnosis, procedures and outcomes in the care of trauma patients ${ }^{5}$. However, trauma registries require a significant financial investment, 1 registrar for every 500-700 annual trauma patients, a cost of approximately \$100140 per patient.

The All Patient Refined Diagnosis Related Groups (APR DRG) classification system groups the diagnoses and care provided during a hospitalization to bundle the reimbursement into similar groups. The APR DRG system classifies patients into like categories and further stratifies them by severity of illness and risk of mortality ${ }^{4}$. The APR DRG system can be used for resource allocation, financial planning, and quality assurance. Studies evaluating the accuracy of the APR DRG classification system for reporting clinical outcomes have been done outside of trauma, but to our knowledge this is the first to compare the trauma registry to the APR DRG ${ }^{6,9-}$ 13.

We sought to determine whether the APR DRG classification system could be used as a surrogate for the trauma registry and estimated the effects of those discrepancies on utilization and program evaluation. We hypothesized that the APR DRG is less accurate in identifying trauma patients from an administrative database than from the trauma registry. While APR DRGs might be limited in their ability to identify all patients, we additionally hypothesized that they may be able to accurately represent the trauma population allowing the hospital system to answer questions which are required in order to maintain trauma center verification. 


\section{Methods}

This is a retrospective study including trauma patients from a single Level 1 Pediatric Trauma Center from 01/2012 to 12/2013. Administrative data including diagnoses, procedures, and APR DRG classification derived from the discharge summaries were obtained from the Pediatric Health Information System (PHIS) database. The database is operated by the Children's Hospital Association and contains administrative and financial details for more than 6 million patient cases from 45 children’s hospitals. For this study the hospital administrative system could have been used but we sought to evaluate the feasibility of a similar evaluation across multiple hospitals.

Discharges from the administrative systems were identified if they had one of 13 specific trauma-specific APR DRG ${ }^{4}, 14$. The codes selected from the administrative data set were selected due to being specific for traumatic injuries. Other APR DRG codes may have included some trauma patients, but would not have been limited exclusively to trauma patients. The traumaspecific APR DRGs were:

\section{Surgical APR DRGs}

- APR DRG 20, Craniotomy for Trauma

- APR DRG 308, Hip \& Femur Procedures for Trauma Except Joint Replacement

- APR DRG 910, Craniotomy for Multiple Significant Trauma

- APR DRG 911, Extensive Abdominal/Thoracic Procedures for Multiple Significant Trauma

- APR DRG 912, Musculoskeletal \& Other Procedures for Multiple Significant Trauma

- APR DRG 711, Post-op, Post-Trauma, Other Device Infections with OR Procedures

\section{Medical APR DRGs}

- APR DRG 40, Spinal Disorders \& Injury

- APR DRG 55, Head Trauma with Coma >1 Hour or Hemorrhage

- APR DRG 56, Brain Contusion/Laceration \& Complicated Skull Fracture, Coma $<1 \mathrm{Hr}$ or no Coma

- APR DRG 57, Concussion, Closed Skull Fractures NOS, Uncomplicated Intracranial Injury, Coma $<1$ Hour or No Coma

- $\quad$ APR DRG 135, Major Chest \& Respiratory Trauma 
- $\quad$ APR DRG 384, Contusion, Open Wound \& Other Trauma to Skin \& Subcutaneous Tissue

- $\quad$ APR DRG 930, Multiple Significant Trauma without OR Procedure

Demographic and clinical characteristics included age, gender, hospital length of stay (LOS), the use of intensive care nursing, primary procedures, primary diagnosis and discharge disposition were abstracted from administrative data. Additional patient characteristics and outcome data were abstracted from the trauma registry. All patients in the trauma registry were assumed to have an admitting diagnosis of trauma. Only data from patients who were admitted as inpatients or admitted as observation to the hospital were analyzed. Patients discharged from the emergency department and emergency department deaths were excluded.

The patients identified by trauma-specific APR DRGs were directly matched with the patients in the trauma registry by medical record number (MRN), financial identification number (FIN) and dates of service. Patients found in both databases were considered matched. All patient encounters that were not "matched" were reviewed by a trauma registrar. Burns were excluded in the pediatric centers trauma registry and trauma-specific APR DRGs.

The following variables were compared to evaluate the level of agreement between the administrative database and the trauma registry: overall agreement, severity of illness/injury, ICU utilization, surgical utilization, head injury, simple fractures, and abdominal trauma. Table 1 shows the definitions that were used for comparison purposes between the two datasets. There is no common severity of illness/injury measure in both administrative and registry data.

Discharges from administrative data can be stratified using the severity of illness algorithms in the APR DRG system. This system relies on coded diagnoses, procedures, and is adjusted for specific demographics ${ }^{2,4}$. The trauma registry used the Injury Severity Scoring (ISS) system. Both of these system categorized patients from 1-4 with 1 being the least severe and 4 being the 
most severe. ISS ranges categorization included: 1) minor injuries, classified ISS score of 0-9, 2) moderate injuries, ISS score of 10-15; 3) serious injuries, ISS score of 16-24; and 4) critical injuries, ISS score of 25-75. These scoring systems are not equivalent and this study did not attempt to compare patient agreement between each systems. Because of the discrepancies in patient identification of each system we used these systems to compare how the resource utilization of each population might be described using different data sources.

Comparisons between the two datasets were descriptive because the datasets are very different. Examples include the ranges of severity as well as ICU length of stay. ICU stays are recorded in days in the trauma registry versus midnight census in the administrative data. This analysis sought to describe the aggregate characteristics of the populations defined by a traumaspecific APR DRG and the population defined by the trauma registry. Due to the overlap in these two populations statistical comparisons were not possible. For the trauma registry records that did not have a trauma-specific APR DRG, we descriptively looked at the APR DRG categories.

\section{Results}

\section{Overall Agreement of the Population and Severity of Illness}

There were 1942 trauma registry patients admitted during 2012-2013 and 1004 administrative records were identified with trauma-specific APR DRGs during the study period. Forty-three percent (835/1942) of registry records had an associated trauma-specific APR DRG

and were considered matched (Figure 1). The kappa value for overall agreement was -0.025 (CI -0.036 to -0.015 ) suggesting the trauma-specific APR DRG agree slightly less than $50 \%$ of the time with the trauma registry. Trauma-specific APR DRG codes missed 57.0\% (1107/1942) of the trauma patients admitted to the hospital that were included in the trauma registry. Of the 1942 trauma registry records, 835 had an associated trauma-specific APR DRG. These codes 
have a 43\% sensitivity for appropriately identifying trauma patients. There were 169 (16.8\%, 169/1004) trauma-specific APR DRG records that were not captured in the trauma registry. Almost half of the patients $(83 / 169,49.1 \%)$ were not seen at the pediatric trauma center but at the adult trauma center within the same hospital system which were misidentified in the administrative data. Seventy-six patients (43.8\%) did not meet the trauma registry inclusion criteria, which are similar to the National Trauma Databank inclusion criteria. Examples of patients that did not meet registry inclusion criteria therefore not included in the registry sample were patients admitted for foreign body removal, patients with injuries over 30 days old, and patients with chronic injuries. Specificity was not calculated because of the inability to get true numbers of the entire population at risk. Because reimbursement is different for patients with an inpatient encounter versus observation/ambulatory surgery encounters, it can be difficult to get accurate case counts from administrative data systems that include multiple encounter types. Patients with all types of encounters were included in the trauma registry.

There were 25 cases identified with a trauma-specific APR DRG that should have been included in the trauma registry. These 25 (1.3\% of the total trauma population) patients were subsequently placed into the trauma registry; however they were not included in the remainder of the calculations.

The trauma-specific APR DRG definition did not identify the minor injuries very well compared to the trauma registry. As table 2 demonstrates, the trauma registry includes more minor injuries compared to trauma-specific APR DRG codes (78.8\% and 57.6\% respectively). However, the matched trauma-specific APR DRG patients had $17.9 \%$ of patients classified in serious and critical injury categories compared to the trauma registry that had only $9.0 \%$ of registry patients in comparable severity levels. 


\section{$\underline{\text { Trauma Care Utilization }}$}

ICU Care

Over two-thirds of registry patients requiring ICU care had a trauma-specific APR DRG code (68.8\%, 203/295). As seen in table 2, the severity of illness distribution is similar. However, twenty-five patients in the critical injury category did not have a trauma-specific APR DRG code. Mortality proportions are also similar among ICU patients in the administrative data compared to the trauma registry, which were $6.9 \%$ and $6.1 \%$, respectively.

\section{Surgical Care}

Only $20.4 \%$ of registry patients requiring operative management had associated traumaspecific APR DRGs (177/864). $80.0 \%$ of trauma registry patients who needed some type of surgical utilization in the operating room were classified in the minor injury category versus 23.7\% of patients with a trauma-specific APR DRG (See Table 2).

\section{$\underline{\text { Injury Categories }}$}

\section{Head Injuries}

The trauma-specific APR DRG data captured 73.0\% of the head injuries identified in the trauma registry (515/705) (Table 2). The distribution of severity categories between the two data sets was similar except for the critical injury category. The trauma registry had $7.7 \%$ of patients within the critical injury category while the trauma-specific APR DRG had 3.5\% of patients with a category of critical injury. The proportion of head injury patients with an ICU stay was almost identical between the two datasets as well as mortality and median hospital length of stay. The percentage of craniotomies in the matched trauma-specific APR DRG data was $6.8 \%$ of patients while in the trauma registry there were $7.5 \%$ of patients with a craniotomy procedure.

\section{Simple Fractures}


Orthopedic injuries were underreported in the administrative data when using traumaspecific APR DRG codes. Extremity fractures with a trauma-specific APR DRG captured 12.5\% $(84 / 672)$ of the extremity fractures found in the trauma registry. The administrative data captured 2 upper extremity fractures and 95 lower extremity fractures, of which 91 were femur fractures. However, in the trauma registry during the same time period there were 268 lower extremity fractures, 391 upper extremity fractures, and 13 patients who had both an upper and lower extremity fracture. The primary procedure of the trauma-specific APR DRG data captured 96 orthopedic operative procedures versus 570 in the trauma registry.

\section{Abdominal Injuries}

Abdominal injuries were not identified easily by the trauma-specific APR DRG codes. The trauma code most related to abdominal injuries that involved a procedure was APR DRG 911: Extensive Abdominal/Thoracic Procedures for Multiple Significant Trauma, and only captured 3 patients. In the trauma registry, there were 153 patients with some type of abdominal injury. The trauma registry had 8 patients that had an abdominal operative procedure during the time period. One of the 8 patients in the trauma registry that had a splenic surgical procedure was identified in the trauma-specific APR DRG data. The trauma registry data and the traumaspecific APR DRG data both captured 2 patients with surgical procedures on the kidney.

Non-trauma-specific APR DRG codes

There were 1107 trauma registry records that did not have a trauma- specific APR DRG. The largest category of trauma registry patients without a trauma-specific APR DRG was the patient with some type of fracture. Table 3 shows the top APR DRG categories for the trauma registry records that did not have a trauma-specific APR DRG. There were 575 patients with some type of extremity fracture that were identified in the trauma registry. Many of the patients 
had a fracture type APR DRG code but it was not trauma-specific. Similarly, the 153 trauma registry patients with abdominal injuries who did not have a trauma-specific APR DRG code, were coded under multiple non-traumatic codes such as "Other disorders of the liver, Hepatic coma \& other major acute liver disorders and Other anemia \& disorders of blood and blood forming organs.”

\section{Discussion:}

Trauma-specific APR DRGs appear to only capture a fraction of the entire trauma population and the distribution was skewed towards more severely ill patients. The majority of patients captured in the trauma registry were classified into non-trauma-specific APR DRGs in administrative data systems. These discrepancies in total population and distribution of illness limit the ability of administrative data to accurately evaluate trauma programs. The APR DRG classification is determined by the principal diagnosis coded by a medical biller; as an example, 5 of the patients in the trauma registry who had a traumatic subdural head and craniotomy procedure were classified under APR DRG 21: Craniotomy except for trauma. Medical coding personnel do not have formal trauma training and may not differentiate a traumatic versus nontraumatic head injury. Additionally administrative billing coders are only able to utilize more limited documentation sources. The billing coder can only use the physician and physician extender notes (Physician assistant or Nurse Practitioner) and has to interpret specified diagnoses and procedures. On the other hand, the trauma registrar who abstracts data can utilize any medical notes, autopsy results, or other relevant clinical data.

Trauma centers are now required to complete risk-based adjustment of outcomes. The APR DRG severity of illness categories were created for risk-adjusted resource allocation, reimbursement, and financial planning. The have also been used for risk-adjustment in analyzing 
patient outcomes. Few studies have evaluated the accuracy of these measures when used for risk adjustment in clinical research or quality reporting within trauma programs. Just as Pasquali et. al. found with cardiac patients, using the risk of mortality and severity of illness scores for risk adjustment in clinical research and quality reporting would be inaccurate since over half of trauma patients are not classified into trauma-specific APR DRGs ${ }^{6}$. More work is needed to determine which, if any, APR DRG codes a hospital could utilize to capture more of the trauma population.

Even though the trauma-specific APR DRGs only captured a relatively small fraction of trauma patients in the trauma registry, the administrative data could be a mechanism to determine if any patients were missed in the trauma registry. We were able to find 25 patients who were inadvertently excluded from the trauma registry. Hospital-specific (and by extension the national registry) benefits from multiple back-up systems to properly identify trauma patients becoming more accurate and robust.

We found that the trauma-specific APR DRGs were most similar to trauma registry data when comparing patients who utilized the intensive care unit or had a head injury. Over twothirds of trauma patients utilizing the ICU were captured with trauma-specific APR DRG coding and almost three-quarters of the head injury patients were found with trauma-specific APR DRG codes. Both categories were able to accurately code mortality and generally represented this subpopulation. However, there were patients within the critical injury category that were not captured by the trauma-specific APR DRGs. One reason could be that national coding rules dictate that certain billing codes be utilized before any other codes in a hierarchical manner (i.e., tracheostomy or ventricular shunt). 
The American College of Surgeons mandates that a general surgeon must respond to critically injured patients within 15 minutes of patient arrival in order to remain a Level 1 Trauma Center ${ }^{8}$. Besides the general surgeon, surgical staff must be available for any of the critically injured patients who deteriorate and need an operative intervention. However, if administrators are only using the trauma-specific APR DRG data, general surgeons' patient numbers would be under-estimated. The data demonstrated that abdominal injuries were not captured well compared to the actual numbers found in the trauma registry. Orthopedic trauma would also be severely under estimated with trauma-specific APR DRG data. There is only one trauma-specific APR DRG code for fractures which severely limits which traumatic fractures are captured by the APR DRG coding system. However, neurosurgical utilization may be reasonably estimated with the APR DRG data on head injuries.

Overall operative utilization was not well captured with the administrative data. Only 177 patients with a matched trauma-specific APR DRG code had a primary procedure indicating an operative encounter. This represented $20 \%$ of the entire 864 patients with a surgical procedure in the trauma registry. In order to ensure that there are adequate operating room resources and personnel available for trauma patients needing operative intervention, as well as anesthesia and surgical specialty coverage, it is imperative to have accurate case volume data. Relying on administrative data alone, hospital administrators would conclude that the number of trauma patients going to the operating room over a two-year period would not be significant enough to justify a separate trauma operating room. However, the trauma registry number is vastly different and is indicative of specific trauma OR room utilization. In this regard, the trauma registry is necessary to provide justification for ongoing surgical support. 
The multiple significant trauma without OR Procedure APR DRG code combines polytrauma data into one code based upon the diagnosis. This code may be useful for billing and reimbursement purposes, but for clinical research the codes are nonspecific and therefore difficult to categorize the type of injury for trauma statistics. For example, there were 20 patients that had head injuries based off of ICD-9 codes, however, they were placed into the APR DRG category 930: Multiple significant traumas without OR procedure and 912: Musculoskeletal and other procedures for multiple significant traumas, which does not have a specific head injury indicator. The same is true for simple fractures. Twelve patients were not included in the orthopedic simple fracture group because the APR DRG category was 912 (Musculoskeleltal and other procedures for multiple significant trauma). From a clinical research perspective, if only the APR DRG category was used, one would never be able to know the specific type of injury. Having this injury-specific information is very important to evaluate program outcomes and for administrators in order to staff hospitals with appropriate personnel and equipment.

One of the top five mechanisms of injury at our pediatric trauma center is non-accidental trauma (NAT). Twenty patients in this dataset did not have a trauma-specific APR DRG even though the primary diagnosis was NAT. The APR DRG classification was either classified under 815: Other injury, poisoning \& toxic effect diagnosis or 950: extensive procedures unrelated to principal diagnosis. Of the 20 patients with a primary diagnosis of NAT, 18 had an APR DRG severity of 3 or 4 and all had stays in the ICU. These patients are resource intensive for trauma surgeons and pose a significant problem when using the APR DRG classification system. Again, when administrators are trying to determine resources for staffing in the intensive care unit or evaluating different programs at a hospital, the current APR DRG codes would underestimate the clinical effects of NAT. 


\section{Limitations}

This study has several limitations. First, this is only a descriptive study. The two datasets were developed for different purposes and comparisons were made as closely as could be approximated. As a result, no true statistical comparisons were utilized in this study. Second, in order to look at overall severity we utilized the APR DRG severity of illness levels and ISS score ranges to come up with categories of minor, mild, serious and critical. Comorbidities and complications are calculated into the APR DRG severity levels whereas ISS scores are only anatomically based. Comorbidities and complications are captured separately in the trauma registry. Therefore, this is not a true equivalence in comparing severity of illness. Potentially we could test the agreement between ISS and APR DRG Severity of Illness by looking at all admissions. Perhaps the APR DRG severity of illness codes could complement the trauma registry data. Lastly, coding hierarchy could have classified severely injured trauma patients into different APR DRG billing categories. An example is the NAT case that was categorized under APR DRG 950: Extensive procedure unrelated to principal diagnosis. If the APR DRG data was being used as a surrogate for trauma data, many trauma cases would be excluded from the numbers because the code is not trauma-specific.

\section{Conclusions}

While the administrative data is attractive to use for outcomes because it is readily available, collected on all patients, and there is a low cost for data entry, we have shown that there are significant gaps when identifying trauma patients. APR DRG administrative data have significant short comings with regard to properly identifying trauma patients and should not be used as the only data source for evaluating the needs of a trauma program. However, the APR DRG could be used by trauma registries to ensure that the registry is as complete as possible, 
though the results from this source are likely to be low. The trauma registry continues to be the best tool to assist hospital administrators when evaluating hospital or operating room utilization and quality measures related to trauma. Trauma surgeons need to understand both databases in order to articulate to hospital administrators the differences between the two databases. 


\section{References}

1. Parnell AS, Shults J, Gaynor JW, Leonard MB, Dai D, Feudtner C. Accuracy of the All Patient Refined Diagnosis Related Groups Classification System in congenital heart surgery. Ann Thorac Surg. 2014;97:641-650.

2. Riley G. Administrative and claims records as sources of health care cost data. Med Care. 2009; 47:S51-S55.

3. Ambroggio LV, Shah SS. Administrative data: expanding the infrastructure for pediatric research. J Pediatr. 2013;162:681-684.

4. Averill R, Goldfield N, Hughes JS, Bonazelli J, McCullough EC, Mullin R, et all. 3M APR DRG Classification System. 31 $1^{\text {st }}$ ed. Wallingford, Connecticut: Agency for Healthcare Research and Quality; 2013. Available at https://proxy.medlib.iupui.edu/login?qurl=http://www.hcupus.ahrq.gov/db/nation/nis/grp031_aprdrg_meth_ovrview.pdf. Accessed January 15, 2015.

5. Wynn A, Wise M, Wright MJ, Rafaat A, Wang Y, Steeb G, et all. Accuracy of administrative and trauma registry databases. J Trauma. 2001; 51:464-468.

6. Pasquali SK, Peterson ED, Jacobs JP, He X, Li SJ, Jacobs ML, et all. Differential case ascertainment in clinical registry versus administrative data and impact on outcomes assessment for pediatric cardiac operations. Ann Thorac Surg. 2013;95:197-203.

7. Hunt JP, Cherr GS, Hunter C, Wright MJ, Wang Y, Steeb G, et all. Accuracy of administrative data in trauma: splenic injuries as an example. J Trauma. 2000;49:679688.

8. Rotondo MF, Cribari C, Smith RS. Resources for optimal care of the injured patient 2014. Available at 
Https://www.facs.org/ /media/files/quality\%20programs/trauma/vrc\%20resources/resour ces\%20for\%20optimal\%20care\%202014\%20v11.ashx. Accessed April 1, 2015.

9. Benneyworth BD, Rigby M, Heskett M, Carroll A, Nitu M. Using All Patient Refined Diagnosis Related Groups to identify cost-management targets. Pediat Therapeut. 2014; $4: 4$.

10. Baram D, Daroowalla F, Garcia R, Zhang G, Chen JJ, Healy E, et all.. Use of the All Patient Refined-Diagnosis Related Group (APR-DRG) risk of mortality score as a severity adjustor in the medical ICU. Clin Med Circ Respirat Pulm Med. 2008; 2:19-25.

11. Iwashyna TJ, Odden A, Rohde J, Bonham C, Kuhn L, Malani P, et all. Identifying patients with severe sepsis using administrative claims. Medical Care. 2014; 52:e39-e43

12. Seymour CW, Iwashyna TJ, Cooke CR. Managing uncertainty in claims-based sepsis research. Crit Care Med. 2013; 41:1134-1136.

13. Tieder JS, Hall M, Auger KA, Jeradi KE, Myers AL, Rahman SS, et all. Accuracy of administrative billing codes to detect urinary tract infection hospitalizations. Pediatrics. 2011;128:323-330.

14. Benneyworth BD, Bennett WE, Carrol AE. Cross-sectional comparison of critically ill pediatric patients across hospitals with various levels of pediatric care. BMC Research Notes. In Press. 
Table 1: Variable definitions used to compare administrative and registry datasets

\begin{tabular}{|c|c|c|}
\hline Variables & Administrative & Registry Definition \\
\hline Severity of Illness Category & APR DRG SOI (Severity of Illness) & ISS (Injury Severity Score) \\
\hline Hospital LOS & Hospital Full Days & Hospital Full Days \\
\hline ICU LOS & Intensive care nursing charge & Full Days \\
\hline Mortality & Discharge Disposition & $\begin{array}{l}\text { Discharge Status in Registry- } \\
\text { Alive or Dead }\end{array}$ \\
\hline Surgery & Primary Procedure & Operating Room Procedure \\
\hline Head Injury & APR DRG Category 20, 55, 56, 57, 910 & $\begin{array}{l}\text { AAAM AIS coding definition of } \\
\text { Head Injury }\end{array}$ \\
\hline Craniotomy & APR DRG Category 20 or 910 & Surgical Procedure List \\
\hline Simple Extremity Fractures & APR DRG 308 & Across all Diagnosis Codes \\
\hline Abdominal Trauma & APR DRG 911 & Across all Diagnosis Codes \\
\hline
\end{tabular}


Table 2: Comparison between administratively defined trauma patients and the trauma registry patients

\begin{tabular}{|c|c|c|}
\hline & Administrative Data & Trauma Registry \\
\hline Overall Trauma Population(n) & 835 & 1942 \\
\hline \multicolumn{3}{|l|}{ Severity of Illness Mix } \\
\hline Minor & $57.6 \%(481 / 835)$ & $78.8 \%(1530 / 1942)$ \\
\hline Moderate & $24.6 \%(205 / 835)$ & $12.3 \%(239 / 1942)$ \\
\hline Serious & $13.1 \%(109 / 835)$ & $5.4 \% \quad(104 / 1942)$ \\
\hline Critical & $4.8 \% \quad(40 / 835)$ & $3.6 \% \quad(69 / 1942)$ \\
\hline Intensive Care Sub-Population (n) & $203(24.3 \%)$ & $295(15.2 \%)$ \\
\hline \multicolumn{3}{|l|}{ Severity of Illness Mix } \\
\hline Minor & $22.2 \%(45 / 203)$ & $32.2 \%(95 / 295)$ \\
\hline Moderate & $24.1 \%(49 / 203)$ & $25.1 \%(74 / 295)$ \\
\hline Serious & $34.0 \%(69 / 203)$ & $20.7 \%(61 / 295)$ \\
\hline Critical & $19.7 \%(40 / 203)$ & $22.0 \%(65 / 295)$ \\
\hline Median Hospital LOS & 3 days & 5 days \\
\hline Intubated Patients & $36.5 \%(74 / 203)$ & $44 / 4 \%(131 / 295)$ \\
\hline ICU Mortality & $6.9 \% \quad(14 / 203)$ & $6.1 \%(18 / 295)$ \\
\hline Operative Sub-Population (n) & $177(21.2 \%)$ & 864 (44.5\%) \\
\hline \multicolumn{3}{|l|}{ Severity of Illness Mix } \\
\hline Minor & $23.7 \%(42 / 177)$ & $80.0 \%(691 / 864)$ \\
\hline Moderate & $32.8 \%(58 / 177)$ & $10.9 \%(94 / 864)$ \\
\hline Serious & $31.6 \%(56 / 177)$ & $3.6 \% \quad(31 / 864)$ \\
\hline Critical & $11.9 \%(21 / 177)$ & $5.6 \% \quad(48 / 864)$ \\
\hline Head Injury Sub-Population (n) & $515(68.9 \%)$ & $705(36.3 \%)$ \\
\hline \multicolumn{3}{|l|}{ Severity of Illness Mix } \\
\hline Minor & $71.1 \%(36 / 515)$ & $69.3 \%(489 / 705)$ \\
\hline Moderate & $16.9 \%(87 / 515)$ & $15.2 \%(107 / 705)$ \\
\hline Serious & $8.5 \%(44 / 515)$ & $7.8 \% \quad(55 / 705)$ \\
\hline Critical & $3.5 \%(18 / 515)$ & $7.7 \%(54 / 705)$ \\
\hline Craniotomies & $6.8 \%(35 / 515)$ & $7.5 \% \quad(53 / 705)$ \\
\hline Median Hospital LOS & 1 day & 1 day \\
\hline ICU patients & $23.9 \%(123 / 515)$ & $22.6 \%(169 / 705)$ \\
\hline Head Injury Mortality & $2.1 \%(11 / 515)$ & $2.5 \%(18 / 705)$ \\
\hline
\end{tabular}

ICU - Intensive Care Unit, LOS - Length of Stay 
Table 3: Non-trauma specific APR DRG codes for the trauma registry patients.

\begin{tabular}{lll}
\hline & \multicolumn{2}{c}{$\begin{array}{c}\text { Patients with non-trauma- } \\
\text { Specific APR DRG codes } \\
\text { Proportion }\end{array}$} \\
APR DRG Code and Name & $\begin{array}{l}\text { Number } \\
\mathbf{( 1 1 0 7 / 1 9 4 2 )}\end{array}$ & $\mathbf{( 5 7 . 0 \% )}$ \\
\hline 315: Shoulder, upper arm \& forearm procedures & 322 & $29.1 \%$ \\
342: Fractures \& dislocations except femur, pelvis \& back & 75 & $6.8 \%$ \\
340: Fracture of femur & 72 & $6.5 \%$ \\
115: Other ear, nose, mouth, throat \& cranial/facial diagnosis & 55 & $5.0 \%$ \\
815: Other injury, poisoning \& toxic effect diagnosis & 44 & $4.0 \%$ \\
347: Other back \& neck disorders, fractures \& injuries & 35 & $3.2 \%$ \\
73: Eye procedures except orbit & 32 & $2.9 \%$ \\
313: Knee \& lower leg procedures except foot & 29 & $2.6 \%$ \\
663: Other anemia \& disorders of blood \& blood forming organs & 28 & $2.5 \%$ \\
114: Dental and oral diseases \& injury & 21 & $1.9 \%$ \\
351: Other musculoskeletal system \& connective tissue diagnosis & 19 & $1.7 \%$ \\
364: Other skin, subcutaneous tissue \& related procedures & 18 & $1.6 \%$ \\
316: Hand \& wrist procedures & 18 & $1.6 \%$ \\
341: Fracture of pelvis or dislocation of hip & 15 & $1.4 \%$ \\
254: Other digestive system diagnosis & 14 & $1.3 \%$ \\
283: Other disorders of the liver & 13 & $1.2 \%$ \\
82: Eye disorders except major infections & 12 & $1.1 \%$ \\
92: Facial bone procedures except major cranial/facial bone procedures & 10 & $0.9 \%$ \\
Other & 209 & $18.9 \%$ \\
No APR DRG designated & 66 & $6.0 \%$ \\
\hline APR DRG: All Pationt
\end{tabular}

APR DRG: All Patient Refined Diagnosis Related Group 
Figure 1: Overall agreement between the trauma registry ( $N=1942)$ and the administrative data $(\mathrm{N}=1004)$. Only $43 \%(835 / 1942)$ of the trauma registry records had an associated trauma-specific APR DRG in the administrative data. Of the patients in the pink section not found in the trauma registry, only $14.8 \%$ (25/169) should have been included in the trauma registry. These 25 patients represented only $1.3 \%$ of the total trauma population when added back into the registry $(25 / 1967)$.

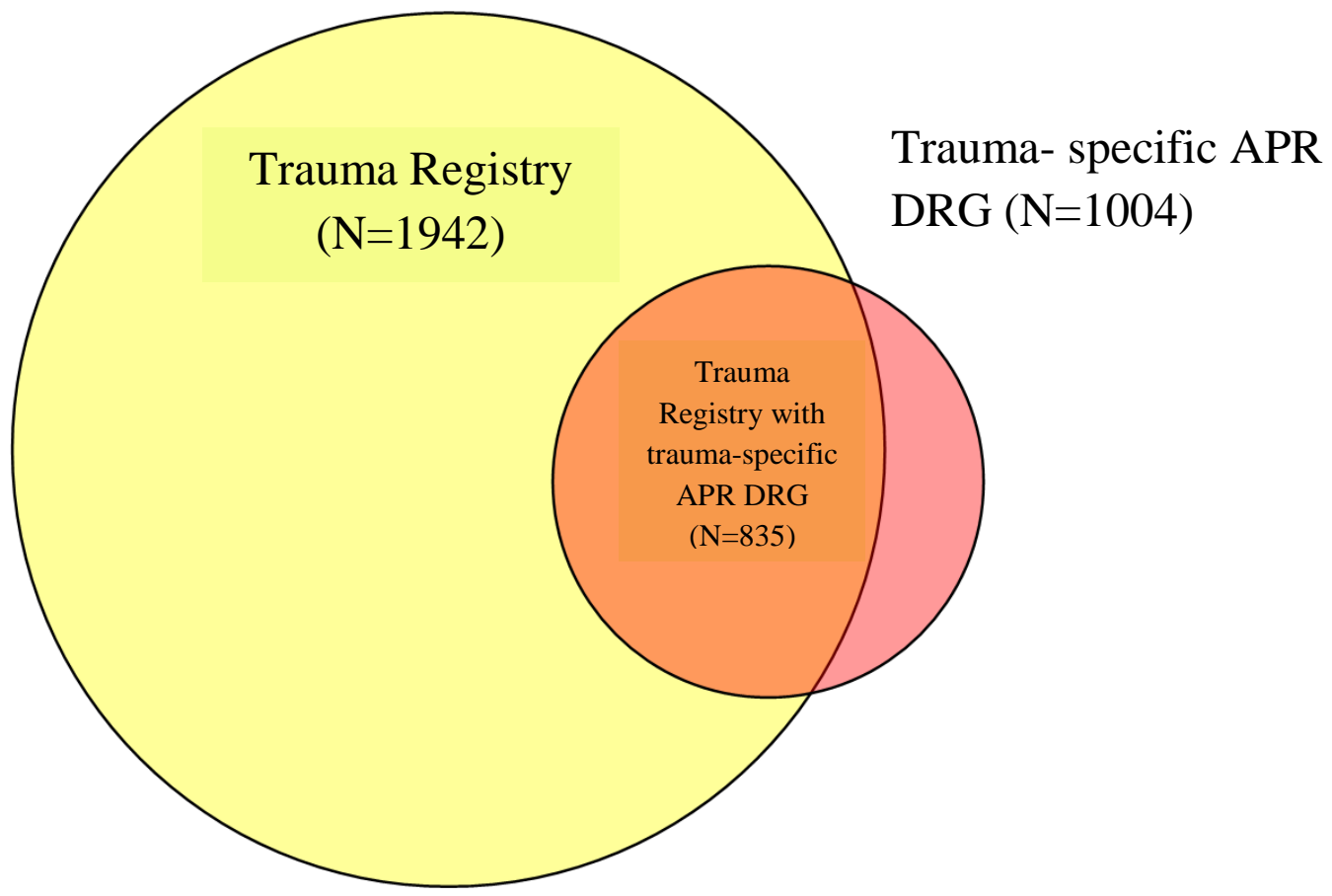

Trauma Registry Records without a trauma-specific APR DRG $\square$ Matched trauma registry records with administrative trauma specific APR DRG data

Administrative data with trauma-specific APR DRGs not included in the registry. 